\title{
BOUNDARY HALF-STRIPS AND THE STRONG CHIP*
}

\author{
EMIL ERNST ${ }^{\dagger}$ AND MICHEL THÉRA ${ }^{\ddagger}$
}

\begin{abstract}
When the subdifferential sum rule formula holds for the indicator functions $\iota_{C}$ and $\iota_{D}$ of two closed convex sets $C$ and $D$ of a locally convex space $X$, the pair $(C, D)$ is said to have the strong conical hull intersection property (the strong CHIP). The specification of a well-known theorem due to Moreau to the case of the support functionals $\sigma_{C}$ and $\sigma_{D}$ subsumes the fact that the pair $(C, D)$ has the strong CHIP whenever the inf-convolution of $\sigma_{C}$ and $\sigma_{D}$ is exact. In this article we prove, in the setting of Euclidean spaces, that if the pair $(C, D)$ has the strong CHIP while the boundary of $C$ does not contain any half-strip, then the inf-convolution of $\sigma_{C}$ and $\sigma_{D}$ is exact. Moreover, when the boundary of a closed and convex set $C$ does contain a half-strip, it is possible to find a closed and convex set $D$ such that the pair $(C, D)$ has the strong CHIP while the inf-convolution of $\sigma_{C}$ and $\sigma_{D}$ is not exact. The validity of the converse of Moreau's theorem in Euclidean spaces is thus associated to the absence of half-strips within the boundary of concerned convex sets.
\end{abstract}

Key words. strong conical hull intersection property, convex programming with convex inequalities, Euclidean space, exact infimal convolution, qualification conditions

AMS subject classifications. 90C46, 90C51, 46N10, 49K40

DOI. $10.1137 / 060658047$

1. Introduction. This study concerns an application of a geometrical notion called the strong conical hull intersection property (strong CHIP) introduced by Deutsch, $\mathrm{Li}$, and Swetits (see [6]). We say that the pair $(C, D)$ of closed and convex subsets of some locally convex space $X$ has the strong CHIP if the subdifferential of the sum and sum of the subdifferentials of their indicator functions coincide:

$$
\partial\left(\iota_{C}+\iota_{D}\right)=\partial \iota_{C}+\partial \iota_{D}
$$

As customary, $\iota_{A}$ is the indicator function of a subset $A$ of $X$ and is defined by $\iota_{A}(x)=0$ if $x \in A$, and $\iota_{A}(x)=+\infty$ otherwise. We also recall that the convex subdifferential is an operator from $X$ into the topological dual $X^{\star}$ of $X$, which assigns to each extended-real-valued mapping $\Phi$ on $X$ a set-valued operator between $X$ and $X^{\star}$ defined by

$$
\partial \Phi\left(x_{0}\right)=\left\{x^{\star} \in X^{*}:\left\langle x-x_{0}, x^{\star}\right\rangle+\Phi\left(x_{0}\right) \leq \Phi(x) \quad \forall x \in X\right\},
$$

where $\langle\cdot, \cdot\rangle: X \times X^{\star} \rightarrow R$ is the duality pairing between $X$ and $X^{\star}$.

Recalling that the normal cone $N_{C}(x)$ at $x \in X$ to a closed convex set $C$ of $X$ is the set $\partial \iota_{C}(x)$, the strong CHIP for the pair $(C, D)$ amounts to saying that

$$
N_{C \cap D}(x)=N_{C}(x)+N_{D}(x) \quad \forall x \in C \cap D ;
$$

i.e., every normal direction to $C \cap D$ at some point $x$ can be expressed as the sum of normal directions at $x$ to $C$ and $D$.

\footnotetext{
*Received by the editors April 24, 2006; accepted for publication (in revised form) January 16, 2007; published electronically DATE.

http://www.siam.org/journals/siopt/x-x/65804.html

${ }^{\dagger}$ Aix-Marseille Université, EA2596, Marseille, F-13397, France (Emil.Ernst@univ.u-3mrs.fr).

${ }^{\ddagger}$ XLIM, Université de Limoges, 123 Avenue A. Thomas, 87060 Limoges Cedex, France (michel. thera@unilim.fr).
} 
This property is important in convex optimization because when we consider the problem of minimizing a convex functional $\Phi$ on the intersection of two sets $C$ and $D$ which have the strong CHIP, the optimality condition for $\bar{x}$ to be a minimizer becomes

$$
0 \in \partial \Phi(\bar{x})+N_{C}(\bar{x})+N_{D}(\bar{x}) .
$$

In the case of convex differentiable optimization, it becomes

$$
-\nabla f(\bar{x}) \in N_{C}(\bar{x})+N_{D}(\bar{x})
$$

Let us quote only the result proved when $X$ is a Hilbert space by Deutsch (see [4]). It says that the strong CHIP is the weakest constraint qualification under which a minimizer $\bar{x}$ of a convex function $\Phi: C_{1} \cap C_{2} \rightarrow R$ can be characterized using the subdifferential of $\Phi$ at $x$ and the normal cones of $C_{1}$ and $C_{2}$ at $x$.

The existence of conditions ensuring that a pair of closed and convex sets has the strong CHIP is based on a classical result by Moreau ([9, Remarque 10.2]); the result initially published in [8] makes use of another key concept of convex analysis, namely the notion of infimal convolution (inf-convolution). Recall that, if $\Phi$ and $\Psi$ are extended-real-valued lower semicontinuous convex functions over $X$ (this class of functions from now on is denoted by $\Gamma_{0}(X)$ ), the inf-convolution of $\Phi$ and $\Psi$ is the extended real-valued function $\Phi \square \Psi$ defined by

$$
\Phi \square \Psi(x)=\inf _{y \in X}(\Phi(x-y)+\Psi(y)) .
$$

The infimal convolution between $\Phi$ and $\Psi$ is said to be exact if $\Phi \square \Psi \in \Gamma_{0}(X)$ and the infimum is achieved in (1.3) whenever $\Phi \square \Psi(x)<+\infty$.

Let us also recall that given a convex closed set $A$ in $X$, we note $\sigma_{A}: X^{\star} \rightarrow$ $R \cup\{+\infty\}$, the support function of $A$. It is defined by

$$
\sigma_{A}(f)=\sup _{x \in A}\langle x, f\rangle
$$

Using this concept, Moreau's theorem states that the subdifferential sum formula (1.1) holds, provided that the inf-convolution of the support functionals of $C$ and $D$ is exact. Remark that an equivalent way of expressing the exactness of the inf-convolution of the support functionals $\sigma_{C}$ and $\sigma_{D}$ is to say that every linear functional $f \in X^{\star}$ which is bounded above on $C \cap D$ may be expressed as the sum of two linear functionals $f_{1}$ and $f_{2}$, bounded above on $C$ and $D$ respectively, such that

$$
\sup _{x \in C \cap D}\langle x, f\rangle=\sup _{x \in C}\left\langle x, f_{1}\right\rangle+\sup _{x \in D}\left\langle x, f_{2}\right\rangle .
$$

Let us observe that relation (1.2), i.e., the strong CHIP, is equivalent to the following property: Every linear functional $f \in X^{\star}$ which achieves its maximum on $C \cap D$ may be expressed as the sum of two linear functionals $f_{1}$ and $f_{2}$, achieving their maximum on $C$ and $D$, respectively, such that

$$
\max _{x \in(C \cap D)}\langle x, f\rangle=\max _{x \in C}\left\langle x, f_{1}\right\rangle+\max _{x \in D}\left\langle x, f_{2}\right\rangle .
$$

The importance of Moreau's theorem comes from the fact that several very general qualification conditions are known to ensure the exactness of the inf-convolution of support functionals (the reader is referred for further information to the excellent 
articles of Zălinescu [13], Gowda and Teboulle [10], and, respectively, Simons [11], in which he/she may find a clear picture of the topic, as well as self-contained proofs for most of the concerned results).

Accordingly, the result proved by Moreau gives the possibility of systematically specifying every qualification condition as a criterion for the strong CHIP (see for instance [5, Proposition 2.3].)

Let us remark that the exactness of the inf-convolution of the support functionals is stronger than the simple strong CHIP. Indeed, Moreau's condition concerns all the continuous linear functionals bounded above on the intersection $C \cap D$, while the strong CHIP is formulated only in terms of those elements from $X^{\star}$ which achieve their maximum on $C \cap D$. The question is thus raised of the validity of the converse to this theorem.

The converse to Moreau's theorem obviously holds for sets $C$ and $D$ such that every linear and continuous map bounded above on any one of the sets $C \cap D, C$, or $D$ necessarily achieve their maximums on this set. On this ground, a first partial converse of the Moreau result has recently been proved by Bauschke, Borwein, and Li for Hilbert spaces (see [1, Proposition 6.4]); the result was extended to the setting of Banach spaces by Burachik and Jeyakumar [3, Proposition 4.2]. Their result states that, if $C$ and $D$ is a pair of closed and convex cones with the strong CHIP, then the inf-convolution of their support functionals is always exact.

However, it is not necessary to impose to every linear and continuous map which is bounded above on any one of the sets $C \cap D, C$, or $D$, to achieve its maximum on this set, in order to ensure the validity of the converse to the above mentionned Moreau's theorem. It is the aim of this article to clearly define the best conditions under which the converse of Moreau's theorem holds. More precisely, we characterize all the closed and convex subsets $C$ of an Euclidean space $X$ such that the following converse of Moreau's theorem holds: If, for some closed and convex set $D$, the pair $(C, D)$ has the strong CHIP, then the inf-convolution of $\sigma_{C}$ and $\sigma_{D}$ is exact.

Our main result states that the validity of the converse of Moreau's theorem is ensured if and only if the boundary of the closed and convex subset $C$ of the Euclidean space $X$ does not contain any half-strip (by half-strip we mean, as customary, the convex hull of two disjoint and parallel half-lines). Note that the class of closed and convex sets without boundary half-strips is rather large, as it contains - the list is not exhaustive - all the bounded sets, the strictly convex sets, or even the continuous sets in the sense of Gale and Klee - sets such that their support functional is continuous except at the origin (see [7]).

The outline of the paper is as follows. The case of closed and convex sets without boundary half-strips is considered in section 2. We prove (Theorem 2.3) that, if the pair $(C, D)$ has the strong CHIP, and if the boundary of one of the sets, say $C$, does not contain any half-strip, then the inf-convolution of the support functions of $C$ and $D$ must be exact.

The last section is concerned with convex sets which do admit at least one boundary half-strip. If the boundary of a closed and convex set $C$ contains some half-strip, then we give a construction of a closed and convex set $D$ such that the pair $(C, D)$ has the strong CHIP, while the inf-convolution of $\sigma_{C}$ and $\sigma_{D}$ fails to be exact.

2. Convex sets without boundary half-strips. Now let us first collect some conditions ensuring in every reflexive Banach space the validity of the converse of Moreau's theorem. 
Proposition 2.1. Let $C$ and $D$ be a pair of closed and convex subsets of a reflexive Banach space $X$. If $C$ and $D$ have the strong CHIP, and at least one of the following conditions holds:

(i) $C \cap D$ is bounded;

(ii) $C \cap D$ is a flat;

(iii) $C \cap D$ is a half-line,

then the inf-convolution of the support functions of $C$ and $D$ is exact. In other words, the converse of Moreau's theorem is valid.

Proof of Proposition 2.1. We need the following standard convex analysis result.

Lemma 2.2. Let $C$ and $D$ be two closed and convex subsets of a locally convex space $X$, and consider an element $y$ of $X^{\star}$ expressed as the sum $y=y_{1}+y_{2}$ of two normal vectors $y_{1} \in N_{C}(x)$ and $y_{2} \in N_{D}(x)$, for some $x \in C \cap D$. Then the inf-convolution of the support functionals is exact at $y$, that is,

$$
\sigma_{C} \square \sigma_{D}(y)=\sigma_{C}\left(y_{1}\right)+\sigma_{D}\left(y_{2}\right)=\langle x, y\rangle .
$$

Proof of Lemma 2.2. As $y_{1} \in \partial \iota_{C}(x)$ and $y_{2} \in \partial \iota_{D}(x)$, relation $y=y_{1}+y_{2}$ implies that $y \in \partial \iota_{C \cap D}(x)$. Thus

$$
\sigma_{C}\left(y_{1}\right)=\left\langle x, y_{1}\right\rangle, \sigma_{2}\left(y_{2}\right)=\left\langle x, y_{2}\right\rangle, \sigma_{C \cap D}(y)=\langle x, y\rangle,
$$

and hence

$$
\sigma_{C \cap D}(y)=\sigma_{C}\left(y_{1}\right)+\sigma_{D}\left(y_{2}\right) .
$$

Recall that $\sigma_{C \cap D} \leq \sigma_{C} \square \sigma_{D}$, which means that

$$
\sigma_{C \cap D}(y) \leq \sigma_{C} \square \sigma_{D}(y) .
$$

Finally, use the definition of the inf-convolution and the fact that $y=y_{1}+y_{2}$ to deduce that

$$
\sigma_{C} \square \sigma_{D}(y) \leq \sigma_{C}\left(y_{1}\right)+\sigma_{D}\left(y_{2}\right) .
$$

Relation (2.1) follows from relations (2.2), (2.3), and (2.4).

Let us now return to the proof of Proposition 2.1 and consider that case (i) holds; i.e., we suppose that the pair $(C, D)$ has the strong CHIP and $C \cap D$ is bounded.

As, in addition, $X$ is a reflexive Banach space, it is easy to see that, for every $y \in X^{\star}$, there is an $x \in C \cap D$ such that $y \in \partial \iota_{C \cap D}(x)$. The pair $(C, D)$ has the strong CHIP, and thus $y=y_{1}+y_{2}$, for some $y_{1} \in \partial \iota_{C}(x)$ and $y_{2} \in \partial \iota_{D}(x)$; we may therefore apply Lemma 2.2 and deduce that

$$
\sigma_{C} \square \sigma_{D}(y)=\sigma_{C}\left(y_{1}\right)+\sigma_{D}\left(y_{2}\right)=\langle x, y\rangle .
$$

On one hand, from relation (2.5) we observe that the $\Gamma_{0}\left(X^{\star}\right)$-functional $\sigma_{C} \square \sigma_{D}$ is real-valued on $X^{\star}$ and thus, as $X^{\star}$ is a reflexive Banach space, is follows that $\sigma_{C} \square \sigma_{D}$ is continuous. Taking into account that relation (2.5) implies, on the other hand, that the infimum is always attained in the expression of the inf-convolution, we conclude that the inf-convolution of the support functions $\sigma_{C}$ and $\sigma_{D}$ is exact.

Case (ii). Let $L$ be the closed subspace of $X$ parallel to the flat $C \cap D$ (that is, $C \cap D=x_{0}+L$ for every $x_{0} \in C \cap D$ ), and factorize $X$ with respect to $L$.

The quotient space $X / L$, say $\hat{X}$, is again a reflexive Banach space. Since $x_{0}+L \subset$ $C$ for every $x_{0} \in C$, and $x_{0}+L \subset D$ for every $x_{0} \in D$, it follows that $\hat{C}$ and $\hat{D}$, the 
quotients of the sets $C$ and $D$ are closed, and convex subsets of $\hat{X}$; moreover, it is straightforward to prove that the pair $(\hat{C}, \hat{D})$ has the strong CHIP if and only if the same holds for the pair $(C, D)$, and that the inf-convolution of the support functions of $\hat{C}$ and $\hat{D}$ is exact if and only if the inf-convolution of the support functions of $C$ and $D$ is exact.

But the intersection between $\hat{C}$ and $\hat{D}$ reduces to a singleton, and case (ii) is proved by applying the conclusion of case (i) to the pair $(\hat{C}, \hat{D})$.

Case (iii). Set $x_{0}+R_{+} \bar{x}$ for the half-line $C \cap D$. Obviously, when $y \in X^{\star}$ and $\langle\bar{x}, y\rangle \leq 0$ we have $y \in \partial \iota_{C \cap D}\left(x_{0}\right)$. Use the fact that the pair $(C, D)$ has the CHIP to deduce that $y=y_{1}+y_{2}$ for some $y_{1} \in \partial \iota_{C}\left(x_{0}\right)$ and $y_{2} \in \partial \iota_{D}\left(x_{0}\right)$, together with Lemma 2.2, to infer that

$$
\sigma_{C} \square \sigma_{D}(y)=\sigma_{C}\left(y_{1}\right)+\sigma_{D}\left(y_{2}\right)=\left\langle x_{0}, y\right\rangle \quad \forall y \in X^{\star},\langle\bar{x}, y\rangle \leq 0 .
$$

In order to obtain a similar relation for the case $\langle\bar{x}, y\rangle>0$, note that, for every $z \in X^{\star}$ such that $\langle\bar{x}, z\rangle>0$ it holds that $\sigma_{C}(z)=\sigma_{D}(z)=+\infty$. Moreover, the inequality $\langle\bar{x}, z+v\rangle>0$ means that at least one of the inequalities $\langle\bar{x}, z\rangle>0$ and $\langle\bar{x}, v\rangle>0$ holds. Combining these two facts, we deduce that

$$
\sigma_{C}(z)+\sigma_{D}(v)=+\infty \quad \forall z, v \in X^{\star} \text { such that }\langle\bar{x}, z+v\rangle>0,
$$

which means that

$$
\sigma_{C} \square \sigma_{D}(y)=+\infty \quad \forall y \in X^{\star},\langle\bar{x}, y\rangle>0 .
$$

Combining relations (2.6) and (2.7) yields

$$
\sigma_{C} \square \sigma_{D}=\iota_{\left\{y \in X^{\star}:\langle\bar{x}, y\rangle \leq 0\right\}}+\left\langle x_{0}, \cdot\right\rangle,
$$

which means that the inf-convolution of $\sigma_{C}$ and $\sigma_{D}$ is the sum between the indicator function of a half-space and a linear and continuous functional, and clearly belongs to $\Gamma_{0}\left(X^{\star}\right)$. Use once more relation (2.6) to see that the infimum in the expression of the inf-convolution is achieved, and conclude that the inf-convolution of $\sigma_{C}$ and $\sigma_{D}$ is exact.

Apparently, Proposition 2.1 lists three completely disparate conditions, each one being sufficient in its own way for the validity of the converse of Moreau's theorem. The geometric notion of a half-strip, that is, a convex hull of two parallel and disjoint half-lines, allows us to spot a common property of cases (i), (ii), and (iii) in Proposition 2.1 .

TheOREm 2.3. Let $C$ and $D$ two closed and convex subsets of the Euclidean space $X$, and assume that the boundary of the set $C$ does not contain any half-strip. If the pair $(C, D)$ has the strong CHIP, then the inf-convolution of $\sigma_{C}$ and $\sigma_{D}$ is exact (in other words, the converse of Moreau's theorem holds).

Proof of Theorem 2.3. When the intersection $C \cap D$ meets the interior of $C$, we specify the well-known Moreau-Rockafellar internal point condition (see [9, Chap. 6, section 6.8]) to prove that the inf-convolution of the support functionals is exact.

If $C \cap D$ is a part of the boundary of $C$, use - as the boundary of $C$ does not contain any half-strip - the obvious fact that the only closed and convex subsets of an Euclidean space which do not contain any half-strip are the bounded sets, the halflines, and the lines, and completely prove Theorem 2.3 by making use of Proposition 2.1 . 


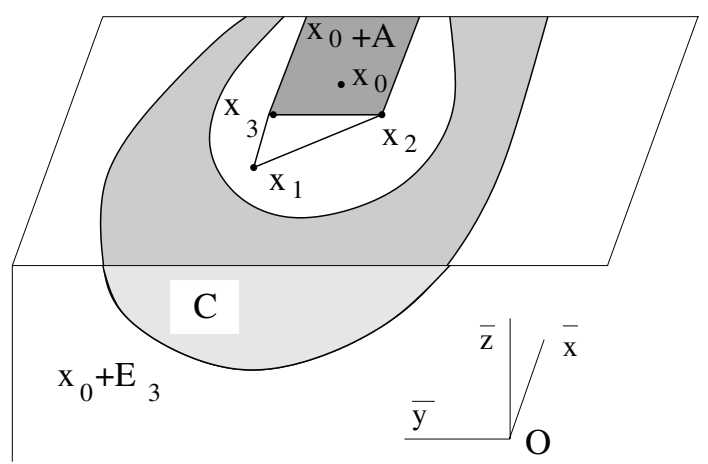

FIG. 3.1. Closed and convex set with a boundary half-strip.

3. Convex sets with boundary half-strips. The following result completes the analysis initiated in Theorem 2.3.

TheOREm 3.1. Let $C$ be a closed and convex subset of the Euclidean space $X$; assume moreover that the boundary of $C$ contains at least one half-strip. Then there is a closed and convex set $D$ such that the pair $(C, D)$ has the CHIP while the infconvolution between $\sigma_{C}$ and $\sigma_{D}$ is not exact.

In other words, the existence of at least one boundary half-strip prevents the converse of Moreau's theorem from holding.

3.1. Construction and properties of the set $D$. Our strategy in this section is to construct the set $D$. The following easy result will be useful. It says that when $X$ is Euclidean, every closed and convex set with a boundary half-strip may be contained within some half-space such that its boundary half-strip lies within the hyperplane which delimits this half-space.

Proposition 3.2. Let $C$ be a closed and convex subset of an Euclidean space $X$ such that its boundary contains a half-strip. Then, there is an orthonormal basis of $X, B=\left\{b_{1}, b_{2}, \ldots, b_{n}\right\}$, a positive parameter $a>0$, and $x_{0}$, an element of $X$, such that

$$
x_{0}+A \subset C \subset x_{0}+E_{3},
$$

where $A$ is the half-strip defined as

$$
A=\left\{x \in X: 0 \leq x \cdot b_{1},-2 a \leq x \cdot b_{2} \leq 2 a, 0=x \cdot b_{i} \quad \forall i \geq 3\right\},
$$

and the half-space $E_{3}$ is given by the relation

$$
E_{3}=\left\{x \in X: x \cdot b_{3} \leq 0\right\} .
$$

Proof of Proposition 3.2. Let $x_{1}, x_{2}, \bar{x}$ in $X$ be such that $\|\bar{x}\|=1$ and the halfstrip spanned by the half-lines $x_{1}+R_{+} \bar{x}$ and $x_{2}+R_{+} \bar{x}$ lies within the boundary of the set $C$. Assume (if necessary after changing $x_{1}$ into $x_{2}$ ) that $x_{2} \cdot \bar{x} \geq x_{1} \cdot \bar{x}$, and set $x_{3}=x_{1}+\left[\left(x_{2}-x_{1}\right) \bar{x}\right] \bar{x}$.

Clearly, $x_{3} \in x_{1}+R_{+} \bar{x}$; as the half-lines $x_{1}+R_{+} \bar{x}$ and $x_{2}+R_{+} \bar{x}$ are disjoint, it follows that $x_{3}$ and $x_{2}$ cannot coincide. Set

$$
a=\frac{\left\|x_{3}-x_{2}\right\|}{4} \quad \text { and } \quad \bar{y}=\frac{x_{3}-x_{2}}{4 a}=\frac{x_{3}-x_{2}}{\left\|x_{3}-x_{2}\right\|} .
$$


Note that

$$
\bar{y} \cdot \bar{x}=\frac{1}{4 a}\left(x_{3} \cdot \bar{x}-x_{2} \cdot \bar{x}\right)=\frac{1}{4 a}\left(x_{1} \cdot \bar{x}+\left(x_{2}-x_{1}\right) \bar{x} \bar{x} \cdot \bar{x}-x_{2} \cdot \bar{x}\right)=0 .
$$

Finally, put

$$
x_{0}=\frac{x_{3}+x_{2}}{2}+a \bar{x}=\frac{1}{2}\left(x_{3}+a \bar{x}\right)+\frac{1}{2}\left(x_{2}+a \bar{x}\right) ;
$$

as $x_{3}+a \bar{x} \in x_{1}+R_{+} \bar{x}$ and $\left(x_{2}+a \bar{x}\right) \in\left(x_{2}+R_{+} \bar{x}\right)$, we deduce that $x_{0}$ belongs to the half-strip spanned by the half-lines $x_{1}+R_{+} \bar{x}$ and $x_{2}+R_{+} \bar{x}$, and thus belongs to the boundary of $C$.

Since $x_{0}$ is a boundary point of a closed and convex subset of an Euclidean space, it is well known that there is some linear mapping $\bar{z} \in X,\|\bar{z}\|=1$, which achieves its maximum on $C$ at $x_{0}$,

$$
\bar{z} \cdot x_{0} \geq \bar{z} \cdot x \quad \forall x \in C
$$

Apply relation (3.2) for $x=x_{0}-a \bar{x}=\frac{x_{3}+x_{2}}{2}$ to deduce that $\bar{z} \cdot \bar{x} \geq 0$, and then for $x=x_{0}+a \bar{x}=\frac{1}{2}\left(x_{3}+2 a \bar{x}\right)+\frac{1}{2}\left(x_{2}+2 a \bar{x}\right)$ to obtain $\bar{z} \cdot \bar{x} \leq 0$ and therefore conclude that

$$
\bar{z} \cdot \bar{x}=0 .
$$

Similarly, put $x_{0}-a \bar{x}-2 a \bar{y}=x_{2}$ for $x$ in relation (3.2), and using also relation (3.3), deduce that $\bar{z} \cdot \bar{y} \geq 0$. Finally, putting $x=x_{0}-a \bar{x}+2 a \bar{y}=x_{3}$ in relation (3.2), and also taking into account relation (3.3), we infer $\bar{z} \cdot \bar{y} \leq 0$, that is,

$$
\bar{z} \cdot \bar{y}=0 .
$$

Relations (3.1), (3.3), and (3.4) prove that it is possible to complete the set $\{\bar{x}, \bar{y}, \bar{z}\}$ up to $B=\left\{b_{1}, b_{2}, \ldots, b_{n}\right\}$, an orthonormal basis of $X$.

The proof of Proposition 3.2 will be completed if we remark that the set $x_{0}+A$ is nothing but the half-strip spanned by the half-lines $\left(x_{3}+a \bar{x}\right)+R_{+} \bar{x}$ and $\left(x_{2}+a \bar{x}\right)+$ $R_{+} \bar{x}$, and thus lies within the boundary of $C$, while relation (3.2) implies that $C$ is a part of the half-space $x_{0}+E_{3}$.

The basis $B$, the parameter $a$, and the element $x_{0}$ thus defined allow us to proceed to the construction of the set $D$. Let us first define the set $F$,

$$
F=\left(P_{1}+S\right) \cap\left(P_{2}+T\right),
$$

where $P_{1} \subset P_{2}$ are the sets bordered by two plane parabolae:

$$
\begin{aligned}
& P_{1}=\left\{x \in X: x \cdot b_{1} \leq-\frac{a\left(x \cdot b_{2}\right)^{2}}{4}, x \cdot b_{i}=0 \quad \forall i \geq 3\right\}, \\
& P_{2}=\left\{x \in X: x \cdot b_{1} \leq-\frac{a\left(x \cdot b_{2}\right)^{2}}{8}, x \cdot b_{i}=0 \quad \forall i \geq 3\right\},
\end{aligned}
$$

$S$ is an orthogonal box in $X$ :

$$
S=\left\{x \in X: x \cdot b_{1} \leq 0,-1 \leq a x \cdot b_{2} \leq 1, x \cdot b_{3} \leq 0\right\},
$$




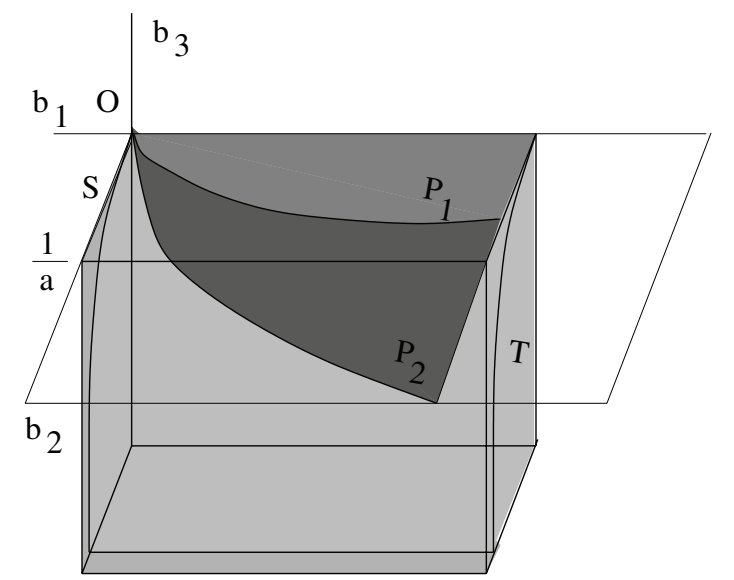

FIG. 3.2. Sets needed in constructing the set D.

$T$ is a closed and convex subset of $S$ :

$$
T=\left\{x \in X: x \cdot b_{1} \leq 0,-1<a x \cdot b_{2}<1, x \cdot b_{3} \leq-\frac{a^{2}\left(x \cdot b_{2}\right)^{2}}{1-a^{2}\left(x \cdot b_{2}\right)^{2}}\right\},
$$

and, as customary, $Z^{\star}$ means the polar set of some subset $Z$ of $X$,

$$
Z^{\star}=\{x \in X: x \cdot y \leq 1 \quad \forall y \in Z\} .
$$

Set now

$$
D=x_{0}+F^{\star}=x_{0}+\left(\left(P_{1}+S\right) \cap\left(P_{2}+T\right)\right)^{\star} .
$$

This definition grants to the set $F$ (and thus $D$ ) several geometrical properties which are crucial for our purpose.

Let us first notice that $F$ is contained in the half-space $E_{1}=\left\{x \in X: x \cdot b_{1} \leq 0\right\}$; accordingly, the half-line $R_{+} b_{1}$ lies within $F^{\star}$, and thus the half-line $x_{0}+R_{+} b_{1}$ is a part of both sets $C$ and $D=x_{0}+F^{\star}$. It follows that

$$
\sigma_{C}(x)=\sigma_{D}(x)=+\infty \quad \forall x \in X \text { such that } x \cdot b_{1}>0 .
$$

Let $x \in X$ be such that $x \cdot b_{1}=0$; if $y$ is such that $y \cdot b_{1} \neq 0$, then either $y \cdot b_{1}>0$ or $(x-y) \cdot b_{1}>0$, so

$$
\sigma_{C}(y)+\sigma_{D}(x-y)=+\infty \quad \forall x \in X, x \cdot b_{1}=0, y \in X, y \cdot b_{1} \neq 0 ;
$$

hence for every element $x \in X$ such that $x \cdot b_{1}=0$ it results that

$$
\sigma_{C} \square \sigma_{D}(x)=\inf _{y \in X, y \cdot b_{1}=0} \sigma_{C}(y)+\sigma_{D}(x-y) .
$$

The hyperplane $L_{1}=\left\{x \in X: x \cdot b_{1}=0\right\}$ thus plays a very important role in computing the inf-convolution of the support functions $\sigma_{C}$ and $\sigma_{D}$. The following lemma describes the intersection between the set $F$ and $L_{1}$; for convenience, we state the result in terms of

$$
\gamma_{F}(x)=\inf _{s>0}\left\{\frac{1}{s}: s x \in F\right\}
$$




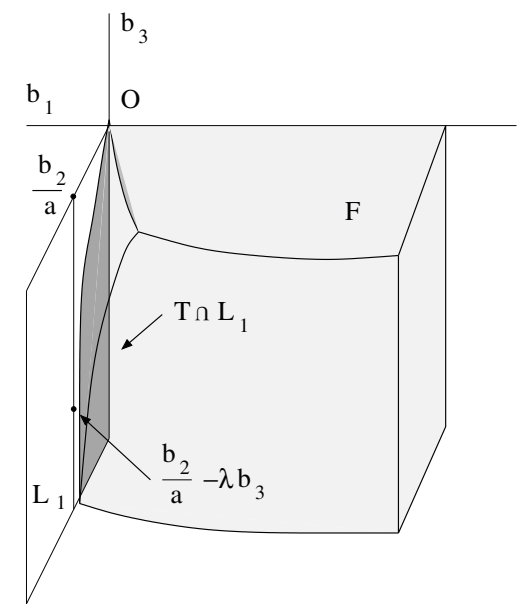

FIG. 3.3. Intersection between $F$ and $L_{1}$.

the gauge function of the set $F$.

Lemma 3.3. The set $F$ is a closed and convex subset of the Euclidean space $X$. Moreover, $F \cap L_{1}=T \cap L_{1}$, and thus

$$
\begin{gathered}
\gamma_{F}(x) \geq a\left|x \cdot b_{2}\right| \quad \forall x \in L_{1}, \\
\gamma_{F}(x)>1 \quad \forall x \in L_{1} \text { such that } a\left|x \cdot b_{2}\right|=1,
\end{gathered}
$$

and

$$
\lim _{\lambda \rightarrow+\infty} \gamma_{F}\left(\frac{b_{2}}{a}-\lambda b_{3}\right)=1 .
$$

Proof of Lemma 3.3. Recall that the sum $Z_{1}+Z_{2}$ of two closed and convex subsets $Z_{1}$ and $Z_{2}$ of an Euclidean space is always convex. This sum is moreover closed, provided that $Z_{1}$ and $-Z_{2}$ do not contain two parallel half-lines (see [12, Corollary 9.1.2] ). This is obviously the case for the pairs of closed and convex sets $P_{1}$ and $S$, as well as $P_{2}$ an $T$, and thus the sets $P_{1}+S$ and $P_{2}+T$ are closed and convex, and the same clearly holds also for the set $F$, which is their intersection.

It has already been noticed that all the sets $P_{1}, P_{2}, S$, and $T$ lay within $E_{1}$; accordingly, the sum of two elements $x_{1}$ and $x_{2}$ from either $P_{1}$ and $S$, or $P_{2}$ and $T$, is contained within the delimiting hyperplane $L_{1}$ if and only if both elements $x_{1}$ and $x_{2}$ belong to $L_{1}$. In other words,

$$
\begin{aligned}
& \left(P_{1}+S\right) \cap L_{1}=\left(P_{1} \cap L_{1}\right)+\left(S \cap L_{1}\right), \\
& \left(P_{2}+T\right) \cap L_{1}=\left(P_{2} \cap L_{1}\right)+\left(T \cap L_{1}\right),
\end{aligned}
$$

and note that $P_{1} \cap L_{1}=P_{2} \cap L_{1}=\{0\}$ to deduce that

$$
\begin{aligned}
F \cap L_{1} & =\left(\left(P_{1}+S\right) \cap L_{1}\right) \cap\left(\left(P_{2}+T\right) \cap L_{1}\right) \\
& =\left(S \cap L_{1}\right) \cap\left(T \cap L_{1}\right)=(S \cap T) \cap L_{1} .
\end{aligned}
$$


Recall that $T \subset S$, and deduce that $F \cap L_{1}=T \cap L_{1}$.

This relation may be used in order to compute the value of $\gamma_{F}(x)$ for elements $x \in L_{1}$, since it obviously holds that

$$
\gamma_{F}(x)=\gamma_{F \cap L_{1}}(x)=\gamma_{T \cap L_{1}}(x) \quad \forall x \in L_{1} .
$$

Use the fact that

$$
T \cap L_{1} \subset S \cap L_{1} \subset M=\left\{x \in X: x \cdot b_{1}=0,-1 \leq a x \cdot b_{2} \leq 1\right\}
$$

to deduce that

$$
\gamma_{T \cap L_{1}}(x) \geq \gamma_{M}(x)=a\left|x \cdot b_{2}\right| \quad \forall x \in L_{1},
$$

that is, relation (3.7).

In order to prove relation (3.8), note that, for every $x \in T \cap L_{1}$ we have $a\left|x \cdot b_{2}\right|<1$. Accordingly, relation $a\left|x \cdot b_{2}\right|=1$ implies that $x \notin T \cap L_{1}$. Let us now use [12, Corollary 9.7.1], which says that $T \cap L_{1}=\left\{y: \gamma_{T \cap L_{1}}(y) \leq 1\right\}$, and deduce that $\gamma_{T \cap L_{1}}(x)>1$.

Finally, if $\lambda \geq 1$, standard computation shows

$$
\frac{\sqrt{4 \lambda^{2}+1}-1}{2 \lambda}\left(\frac{b_{2}}{a}-\lambda b_{3}\right) \in T \cap L_{1} ;
$$

thus

$$
\gamma_{T \cap L_{1}}\left(\frac{b_{2}}{a}-\lambda b_{3}\right) \leq \frac{2 \lambda}{\sqrt{4 \lambda^{2}+1}-1} .
$$

Use relation (3.8) for $x=\left(\frac{b_{2}}{a}-\lambda b_{3}\right)$ to see that

$$
1<\gamma_{T \cap L_{1}}\left(\frac{b_{2}}{a}-\lambda b_{3}\right) ;
$$

relation (3.9) simply comes from relations (3.10) and (3.11).

An important step in proving that the pair of closed and convex sets $(C, D)$ has the strong CHIP is to determine their intersection $C \cap D$.

LEMMA 3.4. It holds that

$$
C \cap D=x_{0}+\left(F+R b_{3}\right)^{\star} .
$$

Proof of Lemma 3.4. Use the fact that $R_{+}\left(-b_{3}\right) \subset T \subset S$ to deduce that $R_{+}\left(-b_{3}\right) \subset F$, and thus that $F^{\star} \subset\left(-E_{3}\right)$. Accordingly, $D \subset x_{0}+\left(-E_{3}\right)$, and as $C \subset x_{0}+E_{3}$, we obtain that

$$
C \cap D \subset\left(x_{0}+E_{3}\right) \cap\left(x_{0}+\left(-E_{3}\right)\right)=x_{0}+L_{3},
$$

where by $L_{3}$ we mean

$$
L_{3}=\left\{x \in X: x \cdot b_{3}=0\right\} .
$$

In other words,

$$
x_{0} \cdot b_{3}=x \cdot b_{3} \quad \forall x \in C \cap D .
$$


Consequently,

$$
C \cap D=C \cap\left(D \cap\left(x_{0}+L_{3}\right)\right)=C \cap\left(x_{0}+\left(F^{\star} \cap L_{3}\right)\right) .
$$

Recall (see [2, Chapter 4, section 1, Corollary of Proposition 3]) that, for every closed and convex sets $A$ and $B$ containing 0 , it holds that $(A \cap B)^{\star}=\overline{\operatorname{co}}\left(A^{\star} \cup B^{\star}\right)$, where $\overline{\mathrm{co}}(A)$ denotes the closed convex hull of the set $A$.

Thus, by using the bipolar theorem (see [2, Chapter 4, section 1, Proposition 3]) applied for the set $F$, and the obvious fact that $L_{3}^{\star}=R b_{3}$, we deduce that

$$
\left(F^{\star} \cap L_{3}\right)^{\star}=\overline{\mathrm{co}}\left(F^{\star \star} \cup L_{3}^{\star}\right)=\overline{\mathrm{co}}\left(F \cup R b_{3}\right) .
$$

It is well known that for every convex set $A$ and flat $W, \overline{\mathrm{co}}(A \cup W)=\overline{\mathrm{co}}(A+W)$. Apply this relation to the convex set $F$ and the one-dimensional flat $R b_{3}$ to prove that $\overline{\mathrm{co}}\left(F \cup R b_{3}\right)=\overline{\mathrm{co}}\left(F+R b_{3}\right)$; by virtue of relation (3.15) it follows that

$$
\left(F^{\star} \cap L_{3}\right)^{\star}=\overline{\mathrm{co}}\left(F+R b_{3}\right) .
$$

Accordingly, $F^{\star} \cap L_{3}=\left(F^{\star} \cap L_{3}\right)^{\star \star}=\left(\overline{\mathrm{co}}\left(F+R b_{3}\right)\right)^{\star}$; as the polar of any set coincides with the polar of its closure, we have

$$
F^{\star} \cap L_{3}=\left(F+R b_{3}\right)^{\star} .
$$

Let us prove that the set $\left(F+R b_{3}\right)^{\star}$ lies within $C$. Indeed, after an easy computation it results that

$$
\begin{aligned}
\overline{\left(T+R b_{3}\right)} & =S+R b_{3} \\
& =N=\left\{x \in X: x \cdot b_{1} \leq 0,-1 \leq a x \cdot b_{2} \leq 1\right\} ;
\end{aligned}
$$

thus $N \subset \overline{\left(F+R b_{3}\right)}$, which means that $\left(F+R b_{3}\right)^{\star} \subset N^{\star}$.

But as

$$
N^{\star}=\left\{x \in X: 0 \leq x \cdot b_{1},-a \leq x \cdot b_{2} \leq a, 0=x \cdot b_{i} \quad \forall i \geq 3\right\},
$$

we have (see Proposition 3.2) $N^{\star} \subset A$, and thus

$$
x_{0}+\left(F+R b_{3}\right)^{\star} \subset\left(x_{0}+N^{\star}\right) \subset\left(x_{0}+A\right) \subset C .
$$

Relation (3.12) follows now from relations (3.14), (3.16), and (3.18).

It thus becomes necessary to determine the sum between the closed and convex set $F$ and the line $R b_{3}$.

LEMMA 3.5. It holds that

$$
\left\{x \in\left(P_{1}+S\right): x \cdot b_{1}<0\right\}+R b_{3} \subset F+R b_{3} \subset\left(P_{1}+S\right)+R b_{3} ;
$$

accordingly,

$$
C \cap D=x_{0}+\left(P_{1}+S+R b_{3}\right)^{\star} .
$$

Moreover, the gauge functions $\gamma_{F}$ and $\gamma_{P_{1}+S+R b_{3}}$ fulfill the following property: For every $x \in X$ such that $x \cdot b_{1}<0$, there is $\theta(x) \geq 0$ such that

$$
\gamma_{P_{1}+S+R b_{3}}(x)=\gamma_{F}\left(x-\theta(x) b_{3}\right) .
$$




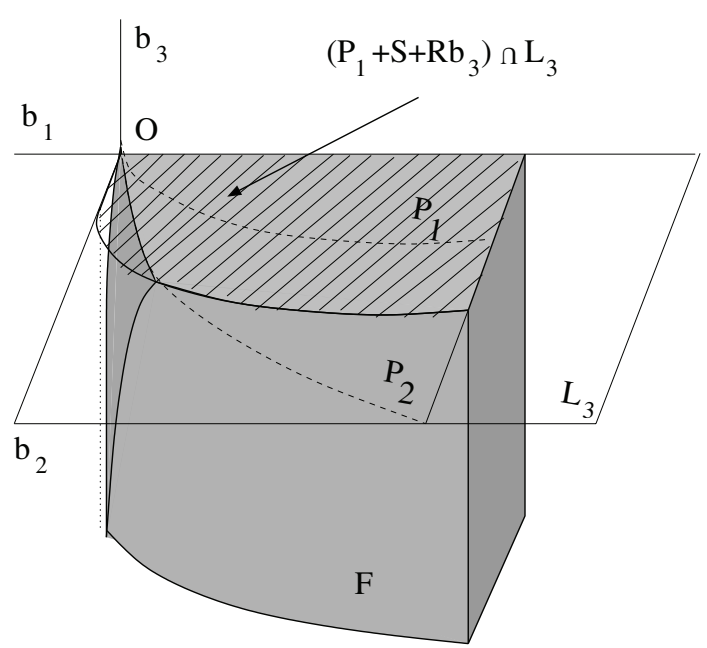

FIG. 3.4. The sum between $F$ and the line $R b_{3}$.

Proof of Lemma 3.5. On the basis of formula (3.17), we claim that $S+R b_{3}$ is a closed and convex set. Moreover, there are no parallel half-lines within $P_{1}$ and $-\left(S+R b_{3}\right)$, so, using again [12, Corollary 9.1.2], we deduce that the set $P_{1}+S+R b_{3}$ is closed and convex.

Let us prove the second inclusion in (3.19). As $F \subset\left(P_{1}+S\right)$, it clearly follows that

$$
F+R b_{3} \subset P_{1}+S+R b_{3}
$$

To establish the first inclusion in relation (3.19), we prove and use the fact that, for every $x \in P_{1}+S$ such that $x \cdot b_{1}<0$, there is $\lambda(x) \geq 0$ such that $\left(x-\lambda(x) b_{3}\right) \in F$.

When $-1<a x \cdot b_{2}<1$, it is easy to see that the value

$$
\lambda(x)=x \cdot b_{3}+\frac{a^{2}\left(x \cdot b_{2}\right)^{2}}{1-a^{2}\left(x \cdot b_{2}\right)^{2}}
$$

does the job. Indeed, the element $y=\left(x \cdot b_{1}\right) b_{1}$ lies within both $P_{1}$ and $P_{2}$, while

$$
z=x-y-\lambda(x) b_{3}=\left(x \cdot b_{2}\right) b_{2}-\frac{a^{2}\left(x \cdot b_{2}\right)^{2}}{1-a^{2}\left(x \cdot b_{2}\right)^{2}} b_{3}+\sum_{i=4}^{n}\left(x \cdot b_{i}\right) b_{i}
$$

is obviously contained in $T$, and thus in $S$. Accordingly,

$$
x-\lambda(x) b_{3}=y+\left(x-y-\lambda(x) b_{3}\right) \in\left(P_{1}+S\right) \cap\left(P_{2}+T\right)=F .
$$

Let $x \in\left(P_{1}+S\right)$ such that $x \cdot b_{1}<0$ and $a\left|x \cdot b_{2}\right| \geq 1$; to fix the ideas, admit that $a x \cdot b_{2} \geq 1$. In order to define $\lambda(x)$ in this case, use the fact that $x$ can be expressed as the sum $x=y+z$ of two elements $y$ and $z$ such that $y \in P_{1}$ and $z \in S$.

As $y \in P_{1}$, it follows that

$$
y \cdot b_{1} \leq-\frac{a\left(y \cdot b_{2}\right)^{2}}{4}
$$


since for every $z \in S$ it holds that $z \cdot b_{1} \leq 0$, we deduce that thus $x \cdot b_{1} \leq y \cdot b_{1}$. We may accordingly infer from relation (3.23) that

$$
x \cdot b_{1} \leq-\frac{a\left(y \cdot b_{2}\right)^{2}}{4} .
$$

Use once more the fact that $z \in S$, to conclude that $-1 \leq a z \cdot b_{2} \leq 1$. Recall that $a x \cdot b_{2} \geq 1$, and deduce that

$$
\left(y \cdot b_{2}\right)^{2}=\left(x \cdot b_{2}-z \cdot b_{2}\right)^{2} \geq\left(x \cdot b_{2}-\frac{1}{a}\right)^{2}
$$

from relation (3.24) and (3.25) it follows that

$$
x \cdot b_{1} \leq-\frac{a\left(x \cdot b_{2}-\frac{1}{a}\right)^{2}}{4} .
$$

Combine the fact that $x \cdot b_{1} \neq 0$ with relation (3.26) and deduce that

$$
x \cdot b_{1}<-\frac{a\left(x \cdot b_{2}-\frac{1}{a}\right)^{2}}{8}
$$

accordingly, for some parameter $\alpha$ such that $0<a \alpha<1$, we have

$$
x \cdot b_{1}<-\frac{a\left(x \cdot b_{2}-\alpha\right)^{2}}{8} .
$$

We can now define $\lambda(x)$ as

$$
\lambda(x)=\frac{a^{2} \alpha^{2}}{1-a^{2} \alpha^{2}} .
$$

Inequality (3.27) proves that the element $\left(x-\alpha b_{2}-\sum_{i=4}^{n}\left(x \cdot b_{i}\right) b_{i}\right)$ belongs to the set $P_{2}$; as obviously

$$
\alpha b_{2}-\frac{a^{2} \alpha^{2}}{1-a^{2} \alpha^{2}} b_{3}+\sum_{i=4}^{n}\left(x \cdot b_{i}\right) b_{i} \in T
$$

we deduce that

$$
\begin{aligned}
x & -\lambda(x) b_{3} \\
& =\left(x-\alpha b_{2}-\sum_{i=4}^{n}\left(x \cdot b_{i}\right) b_{i}\right)+\left(\alpha b_{2}-\frac{a^{2} \alpha^{2}}{1-a^{2} \alpha^{2}} b_{3}+\sum_{i=4}^{n}\left(x \cdot b_{i}\right) b_{i}\right) \\
& \in P_{2}+T .
\end{aligned}
$$

Remark that the case $a x \cdot b_{2} \leq-1$ is similar to the case $a x \cdot b_{2} \geq 1$. Indeed, when $a x \cdot b_{2} \leq-1$, one has

$$
\left(y \cdot b_{2}\right)^{2} \geq\left(x \cdot b_{2}+\frac{1}{a}\right)^{2}
$$

instead of relation (3.25). The parameter $\alpha$ now lies between $-\frac{1}{a}$ and 0 , and fulfills

$$
x \cdot b_{1}<-\frac{a\left(x \cdot b_{2}+\alpha\right)^{2}}{8},
$$


and not relation $(3.26)$.

As in the case $a x \cdot b_{2} \geq 1, x$ is the sum of two elements: one in $P_{2}$, the other in $T$. However, when $a x \cdot b_{2} \leq-1$, the element belonging to $P_{2}$ is $\left(x+\alpha b_{2}-\sum_{i=4}^{n}\left(x \cdot b_{i}\right) b_{i}\right)$, and the one lying in $T$ is $-\alpha b_{2}-\frac{a^{2} \alpha^{2}}{1-a^{2} \alpha^{2}} b_{3}+\sum_{i=4}^{n}\left(x \cdot b_{i}\right) b_{i}$.

Noticing that $S+R_{+}\left(-b_{3}\right)=S$, we have $\left(P_{1}+S+R_{+} b_{3}\right)=\left(P_{1}+S\right)$. Thus, as $x \in P_{1}+S$, we deduce that

$$
x-\lambda b_{3} \in P_{1}+S \quad \forall \lambda \geq 0
$$

from (3.28) and (3.29) it follows that

$$
\left(x-\lambda(x) b_{3}\right) \in\left(P_{1}+S\right) \cap\left(P_{2}+T\right)=F,
$$

and therefore for every $x \in P_{1}+S$ such that $x \cdot b_{1}<0$, there is $\lambda(x) \geq 0$ such that $x-\lambda(x) b_{3} \in F$.

Use this observation to prove that

$$
\left\{x \in P_{1}+S: x \cdot b_{1}<0\right\}+R b_{3} \subset F+R b_{3},
$$

which, together with relation (3.22), yields relation (3.19).

Relation (3.19) implies that the set $P_{1}+S+R b_{3}$ is the closure of the set $F+R b_{3}$. Recalling that the polar of any set coincides with the polar of its closure, we deduce that

$$
\left(F+R b_{3}\right)^{\star}=\left(P_{1}+S+R b_{3}\right)^{\star},
$$

and relation (3.20) follows from formulas (3.12) and (3.30).

It remains to prove relation (3.21). To begin with, notice that, from relation (3.19) it follows that $F \subset P_{1}+S+R b_{3}$, and thus

$$
\gamma_{P_{1}+S+R b_{3}} \leq \gamma_{F}
$$

Let us first prove that $\gamma_{P_{1}+S+R b_{3}}(x)$ is real-valued for every $x \in X$ such that $x \cdot b_{1}<0$. In this respect, note that from relation (3.17) it results that $\{x \in X$ : $\left.x \cdot b_{2}=x \cdot b_{3}=0\right\} \subset S+R b_{3}$, and thus that

$$
P_{1}+\left\{x \in X: x \cdot b_{1}=x \cdot b_{2}=0\right\} \subset\left(P_{1}+S+R b_{3}\right) .
$$

On the other hand, the set $P_{1}+\left\{x \in X: x \cdot b_{1}=x \cdot b_{2}=0\right\}$ contains all the elements $x \in X$ such that $x \cdot b_{1} \leq-\frac{a\left(x \cdot b_{2}\right)^{2}}{4}$. As

$$
\left(-\frac{4 x \cdot b_{1}}{a\left(x \cdot b_{2}\right)^{2}} x\right) \cdot b_{1}=-\frac{4\left(x \cdot b_{1}\right)^{2}}{a\left(x \cdot b_{2}\right)^{2}}=-\frac{a\left(\left(-\frac{4 x \cdot b_{1}}{a\left(x \cdot b_{2}\right)^{2}} x\right) \cdot b_{2}\right)^{2}}{4}
$$

which means that

$$
-\frac{4 x \cdot b_{1}}{a\left(x \cdot b_{2}\right)^{2}} x \in P_{1}+\left\{x \in X: x \cdot b_{1}=x \cdot b_{2}=0\right\} .
$$

Combine the previous relation with formula (3.32) to deduce that

$$
\gamma_{P_{1}+S+R b_{3}}(x) \leq \frac{a\left(x \cdot b_{2}\right)^{2}}{\left|4 x \cdot b_{1}\right|}
$$


Consequently, for every $x \in X$ such that $x \cdot b_{1}<0$ we have $\gamma_{P_{1}+S+R b_{3}}(x)<+\infty$.

Let us first consider the case when $\gamma_{P_{1}+S+R b_{3}}(x)=0$, that is, when $x$ belongs to a ray completely contained in $P_{1}+S+R b_{3}$. Taking into account the definitions of the sets $P_{1}$ and $S$, we have

$$
\left[\gamma_{P_{1}+S+R b_{3}}(x)=0\right] \Leftrightarrow\left[x \cdot b_{1} \leq 0 \text { and } x \cdot b_{2}=0\right] ;
$$

similarly,

$$
\left[\gamma_{F}(x)=0\right] \Leftrightarrow\left[x \cdot b_{1} \leq 0, x \cdot b_{2}=0 \text { and } x \cdot b_{3} \leq 0\right] .
$$

In this case, $\theta(x)=\frac{x \cdot b_{3}+\left|x \cdot b_{3}\right|}{2}$ obviously does the job.

Let us now turn to the case $\gamma_{P_{1}+S+R b_{3}}(x)>0$ and write that

$$
\frac{x}{\gamma_{P_{1}+S+R b_{3}}(x)} \in P_{1}+S+R b_{3} \text {. }
$$

We deduce that there is a $\tilde{\lambda}(x) \in R$ such that

$$
\frac{x}{\gamma_{P_{1}+S+R b_{3}}(x)}-\tilde{\lambda}(x) b_{3} \in P_{1}+S .
$$

Accordingly,

$$
\left(\frac{x}{\gamma_{P_{1}+S+R b_{3}}(x)}-\tilde{\lambda}(x) b_{3}\right)-\lambda\left(\frac{x}{\gamma_{P_{1}+S+R b_{3}}(x)}-\tilde{\lambda}(x) b_{3}\right) b_{3} \in F,
$$

which means that

$$
\begin{gathered}
\gamma_{F}\left(x-\gamma_{P_{1}+S+R b_{3}}(x)\left(\tilde{\lambda}(x)+\lambda\left(\frac{x}{\gamma_{P_{1}+S+R b_{3}}(x)}-\tilde{\lambda}(x) b_{3}\right)\right) b_{3}\right) \\
\leq \gamma_{P_{1}+S+R b_{3}}(x) .
\end{gathered}
$$

Relations (3.31), (3.33) and the obvious fact that

$$
\gamma_{P_{1}+S+R b_{3}}(x)=\gamma_{P_{1}+S+R b_{3}}\left(x+\nu b_{3}\right) \quad \forall \nu \in R
$$

prove relation (3.21) with

$$
\theta(x)=\gamma_{P_{1}+S+R b_{3}}(x)\left(\tilde{\lambda}(x)+\lambda\left(\frac{x}{\gamma_{P_{1}+S+R b_{3}}(x)}-\tilde{\lambda}(x) b_{3}\right)\right),
$$

completing in this way the proof of Lemma 3.5.

3.2. The main result. We claim that the pair of closed and convex sets $C$ and $D$ has the strong CHIP.

Proposition 3.6. The pair of closed and convex subsets $C$ and $D$ of the Euclidean space $X$ has the strong CHIP.

Proof of Proposition 3.6. Let $x_{1} \in C \cap D$ and $y \in \partial \iota_{C \cap D}\left(x_{1}\right), y \neq 0$. Our aim is to express $y$ as the sum of two elements $y_{1}$ and $y_{2}$ from $\partial \iota_{C}\left(x_{1}\right)$ and $\partial \iota_{D}\left(x_{1}\right)$.

Let us first remark that, since $\left(x_{0}+A\right) \subset C \subset\left(x_{0}+E_{3}\right)$ (see Proposition 3.2), it follows that

$$
R_{+} b_{3} \subset \partial \iota_{C}(x)
$$


for every $x \in x_{0}+A$, in particular for every $x \in C \cap D$ (see Lemma 3.4 and relation (3.18)). Similarly, we deduce that

$$
R_{+}\left(-b_{3}\right) \subset \partial \iota_{D}(x)
$$

for every $x \in C \cap D$. The flat $\left\{x \in X: x \cdot b_{i}=0 \forall 1 \leq i \leq 3\right\}$ obviously lies within $T$, and thus in $S$, hence in $F$. Thus $D$ is contained within the flat $x_{0}+\left\{x \in X: x \cdot b_{i}=\right.$ $0 \forall i \geq 4\}$, and we deduce that

$$
\left\{x \in X: x \cdot b_{1}=x \cdot b_{2}=x \cdot b_{3}=0\right\} \subset \partial \iota_{D}(x) \quad \forall x \in D .
$$

From relations (3.34), (3.35), and (3.36) it follows that

$$
\left\{x \in X: x \cdot b_{1}=x \cdot b_{2}=0\right\} \subset\left(\partial \iota_{C}(x)+\partial \iota_{D}(x)\right) \quad \forall x \in(C \cap D) .
$$

We address first the case when $y \cdot b_{1}=0$. A standard computation shows that

$$
\begin{aligned}
& \left(P_{1}+S+R b_{3}\right)^{\star} \\
& =\left\{x \in X: x \cdot b_{1} \geq \frac{\left(x \cdot b_{2}\right)^{2}}{a-\left|x \cdot b_{2}\right|},-a<x \cdot b_{2}<a, x \cdot b_{i}=0 \quad \forall i \geq 3\right\} .
\end{aligned}
$$

From the previous relation it follows that the set $\left(P_{1}+S+R b_{3}\right)^{\star}$ is contained within the plane spanned by $b_{1}$ and $b_{2}$. For every $y \in X$ such that $y \cdot b_{1}=0$ it follows that

$$
x \cdot y=\left(x \cdot b_{2}\right)\left(y \cdot b_{2}\right) \quad \forall x \in\left(P_{1}+S+R b_{3}\right)^{\star} .
$$

The elements $x_{1}$ and $x_{0}$ are both in $C \cap D$; in view of relation (3.20) it appears that $\left(x_{1}-x_{0}\right) \in\left(P_{1}+S+R b_{3}\right)^{\star}$.

From relation $(3.38)$ it follows that $\left|\left(x_{1}-x_{0}\right) \cdot b_{2}\right|<a$. Set

$$
\alpha=\frac{a+\left|\left(x_{1}-x_{0}\right) \cdot b_{2}\right|}{2}, z_{1}=\frac{\alpha^{2}}{a-\alpha} b_{1}-\alpha b_{2}, z_{2}=\frac{\alpha^{2}}{a-\alpha} b_{1}+\alpha b_{2} ;
$$

thus $z_{1}, z_{2} \in\left(P_{1}+S+R b_{3}\right)^{\star}$ and $z_{1} \cdot b_{2}<\left(x_{1}-x_{0}\right) \cdot b_{2}<z_{2} \cdot b_{2}$.

Recall that, as $y \in \partial \iota_{C \cap D}\left(x_{1}\right)$, the linear functional $X \ni x \rightarrow(x \cdot y) \in R$ achieves its maximum on $C \cap D$ at $x_{1}$. Thus, on one hand,

$$
\left(x_{0}+z_{1}\right) \cdot y \leq x_{1} \cdot y,
$$

that is, in view of relation (3.39),

$$
\left(z_{1} \cdot b_{2}\right)\left(y \cdot b_{2}\right) \leq\left(\left(x_{1}-x_{0}\right) \cdot b_{2}\right)\left(y \cdot b_{2}\right)
$$

combine this relation with the fact that $z_{1} \cdot b_{2}<\left(x_{1}-x_{0}\right) \cdot b_{2}$, and deduce that $y \cdot b_{2} \geq 0$. On the other hand,

$$
\left(x_{0}+z_{2}\right) \cdot y \leq x_{1} \cdot y,
$$

that is, once more by virtue of relation (3.39),

$$
\left(z_{2} \cdot b_{2}\right)\left(y \cdot b_{2}\right) \leq\left(\left(x_{1}-x_{0}\right) \cdot b_{2}\right)\left(y \cdot b_{2}\right) ;
$$

in addition, as $\left(x_{1}-x_{0}\right) \cdot b_{2}<z_{2} \cdot b_{2}$, we get $y \cdot b_{2} \leq 0$. 
We may thus conclude that, when $y \cdot b_{1}=0$, it results that $y \cdot b_{2}=0$, and formula (3.37) proves that $y$ is the sum of two elements from $\partial \iota_{C}\left(x_{1}\right)$ and $\partial \iota_{D}\left(x_{1}\right)$.

Consider now the case when $y \cdot b_{1} \neq 0$, which, taking into account the fact that the half-line $\left(x_{0}+R_{+} b_{1}\right)$ is contained (as already remarked) within $C \cap D$, amounts to saying that $y \cdot b_{1}<0$.

It is well known ([12, Theorem 14.5]) that, for every closed and convex set $Z$ containing 0 it holds $\sigma_{Z}=\gamma_{Z^{\star}}$. Use this relation for the set $\left(P_{1}+S+R b_{3}\right)^{\star}$ to obtain

$$
\sigma_{\left(P_{1}+S+R b_{3}\right)^{\star}}=\gamma_{P_{1}+S+R b_{3}}
$$

as (see 3.20) $\left(P_{1}+S+R b_{3}\right)^{\star}=(C \cap D)-x_{0}$, it follows that

$$
\sigma_{(C \cap D)-x_{0}}=\gamma_{P_{1}+S+R b_{3}} .
$$

Similarly,

$$
\sigma_{D-x_{0}}=\gamma_{F}
$$

From relations (3.21), (3.40), and (3.41) it follows that there is some $\theta(y) \geq 0$ such that

$$
\sigma_{(C \cap D)-x_{0}}(y)=\sigma_{D-x_{0}}\left(y-\theta(y) b_{3}\right)
$$

thus

$$
\sigma_{C \cap D}(y)=\sigma_{D}\left(y-\theta(y) b_{3}\right)+\theta(y) x_{0} \cdot b_{3} .
$$

As $y \in \partial \iota_{C \cap D}\left(x_{1}\right)$, it results that $\sigma_{C \cap D}(y)=x_{1} \cdot y$; use relation (3.42) to see that

$$
\sigma_{D}\left(y-\theta(y) b_{3}\right)+\theta(y) x_{0} \cdot b_{3}=x_{1} \cdot y .
$$

Relation (3.13) reads $x_{0} \cdot b_{3}=x_{1} \cdot b_{3}$. Equality (3.43) may thus be stated as

$$
\sigma_{D}\left(y-\theta(y) b_{3}\right)+\theta(y) x_{1} \cdot b_{3}=x_{1} \cdot y,
$$

that is,

$$
\sigma_{D}\left(y-\theta(y) b_{3}\right)=x_{1} \cdot\left(y-\theta(y) b_{3}\right) .
$$

This means that $\left(y-\theta(y) b_{3}\right) \in \partial \iota_{D}\left(x_{1}\right)$.

Recall (see relation (3.34)) that $\lambda b_{3} \in \partial \iota_{C}\left(x_{1}\right)$ for every $\lambda \geq 0$, and express $y$ as $y=\theta(y) b_{3}+\left(y-\theta(y) b_{3}\right)$, that is, the sum of an element from $\partial \iota_{C}\left(x_{1}\right)$ and the sum of another element from $\partial \iota_{D}\left(x_{1}\right)$.

We finally claim that the inf-convolution of the support functionals $\sigma_{C}$ and $\sigma_{D}$ is not exact at $\frac{b_{2}}{a}$, a fact which completes the proof of Theorem 3.1.

Proposition 3.7. It holds that

$$
\sigma_{C} \square \sigma_{D}\left(\frac{b_{2}}{a}\right)=\frac{x_{0} \cdot b_{2}}{a}+1,
$$

while

$$
\sigma_{C}(y)+\sigma_{D}(z)>\frac{x_{0} \cdot b_{2}}{a}+1 \quad \forall y+z=\frac{b_{2}}{a} .
$$


Proof of Proposition 3.7. Use the fact that $\left(x_{0}+2 a b_{2}\right)$ and $\left(x_{0}-2 a b_{2}\right)$ both belong to $x_{0}+A$, and thus to $C$, to deduce that

$$
\sigma_{C}(x) \geq \max \left(\left(x_{0}+2 a b_{2}\right) \cdot x,\left(x_{0}-2 a b_{2}\right) \cdot x\right)=x_{0} \cdot x+2 a\left|x \cdot b_{2}\right| .
$$

From relation (3.7) it follows that

$$
\gamma_{F}(x) \geq a\left|x \cdot b_{2}\right| \quad \forall x \in L_{1}
$$

Relation (3.41) reads $\sigma_{D-x_{0}}=\gamma_{F}$; hence, it results that

$$
\sigma_{D}(x)=x_{0} \cdot x+\gamma_{F}(x)
$$

It follows that

$$
\sigma_{D}(x) \geq x_{0} \cdot x+a\left|x \cdot b_{2}\right| \quad \forall x \in L_{1}
$$

From relations (3.46) and (3.48) it results that

$$
\begin{aligned}
& \sigma_{C}(x)+\sigma_{D}\left(\frac{b_{2}}{a}-x\right) \\
& \geq x_{0} \cdot x+2 a\left|x \cdot b_{2}\right|+x_{0} \cdot\left(\frac{b_{2}}{a}-x\right)+a\left|\left(\frac{b_{2}}{a}-x\right) \cdot b_{2}\right| \\
& \geq \frac{x_{0} \cdot b_{2}}{a}+1+a\left|x \cdot b_{2}\right| \quad \forall x \in X, x \cdot b_{1}=0 .
\end{aligned}
$$

By taking into account relations (3.6) and (3.49) we prove that

$$
\sigma_{C} \square \sigma_{D}\left(\frac{b_{2}}{a}\right) \geq \frac{x_{0} \cdot b_{2}}{a}+1
$$

As $x_{0} \in C \subset\left(x_{0}+E_{3}\right)$, it follows that

$$
\sigma_{C}\left(\lambda b_{3}\right)=\lambda x_{0} \cdot b_{3} \quad \forall \lambda \geq 0 .
$$

Use relation (3.47) to deduce that

$$
\begin{aligned}
\sigma_{C}\left(\lambda b_{3}\right) & +\sigma_{D}\left(\frac{b_{2}}{a}-\lambda b_{3}\right) \\
& =\lambda x_{0} \cdot b_{3}+\frac{x_{0} \cdot b_{2}}{a}-\lambda x_{0} \cdot b_{3}+\gamma_{F}\left(\frac{b_{2}}{a}-\lambda b_{3}\right) .
\end{aligned}
$$

From the previous equality, together with relation (3.9), it yields that

$$
\lim _{\lambda \rightarrow \infty}\left(\sigma_{C}\left(\lambda b_{3}\right)+\sigma_{D}\left(\frac{b_{2}}{a}-\lambda b_{3}\right)\right)=\frac{x_{0} \cdot b_{2}}{a}+1,
$$

which, combined with inequality (3.50), proves relation (3.44).

Finally, let $x \in L_{1}$ be such that $x \cdot b_{2}=0$. Then (see relation (3.8))

$$
\gamma_{F}\left(\frac{b_{2}}{a}-x\right)>1
$$


and, as obviously $\sigma_{C}(x) \geq x_{0} \cdot x$, it results that

$$
\begin{aligned}
\sigma_{C}(x) & +\sigma_{D}\left(\frac{b_{2}}{a}-x\right) \\
& \geq x_{0} \cdot x+\frac{x_{0} \cdot b_{2}}{a}-x_{0} \cdot x+\gamma_{F}\left(\frac{b_{2}}{a}-x\right) \\
& >\frac{x_{0} \cdot b_{2}}{a}+1 \quad \forall x \in X, x \cdot b_{1}=x \cdot b_{2}=0 .
\end{aligned}
$$

Use relation (3.49) to deduce that, for every $x \in X$ such that $x \cdot b_{1}=0$ and $x \cdot b_{2} \neq 0$, it holds that

$$
\sigma_{C}(x)+\sigma_{D}\left(\frac{b_{2}}{a}-x\right)>\frac{x_{0} \cdot b_{2}}{a}+1 .
$$

Relation (3.45) follows from relations (3.5), (3.52), and (3.51).

Acknowledgments. We would like to warmly thank the two anonymous referees. Their careful reading of the paper allowed us to correct a significant number of typos and errors and largely contributed to the final form of the article.

\section{REFERENCES}

[1] H. H. Bauschke, J. M. Borwein, And W. LI, The strong conical hull intersection property, bounded linear regularity, Jameson's property $(G)$, and error bounds in convex optimization, Math. Program. Ser. A., 86 (1999), pp. 135-160.

[2] N. Bourbaki, Eléments de mathématique. XVIII, Actualités Sci. Ind. 1229, Hermann \& Cie, Paris, 1955.

[3] R. S. Burachik and V. Jeyakumar, A simple closure condition for the normal cone intersection formula, Proc. Amer. Math. Soc., 133 (2005), pp. 1741-1748.

[4] F. Deutsch, The role of the strong conical hull intersection property in convex optimization and approximation, in Approximation Theory IX, Innov. Appl. Math., Vanderbilt University Press, Nashville, TN, 1998, pp. 105-112.

[5] F. Deutsch, W. Li, AND J. D. Ward, Best approximation from the intersection of a closed convex set and a polyhedron in Hilbert space, weak Slater conditions, and the strong conical hull intersection property, SIAM J. Optim., 10 (1999), pp. 252-268.

[6] F. Deutsch, W. Li, And J. Swetits, A dual approach to constrained interpolation from a convex subset of Hilbert space, J. Approx. Theory, 90 (1997), pp. 385-414.

[7] D. Gale and V. Klee, Continuous convex sets, Math. Scand., 7 (1959), pp. 370-391.

[8] J. J. Moreau, Étude locale d'une fonctionnelle convexe, Université de Montpellier, Montpellier, France, 1963.

[9] J. J. Moreau, Fonctionnelles convexes, séminaire sur les équations aux dérivées partielles, Collège de France, Paris, 1967.

[10] M. S. Gowda And M. Teboulle, A comparison of constraint qualifications in infinitedimensional convex programming, SIAM J. Control Optim., 28 (1990), pp. 925-935.

[11] S. Simons, Sum theorems for monotone operators and convex functions, Trans. Amer. Math. Soc., 350 (1998), pp. 2953-2972.

[12] R. T. Rockafellar, Convex Analysis, Princeton University Press, Princeton, NJ, 1968.

[13] C. ZăLineSCU, A comparison of constraint qualifications in infinite-dimensional convex programming revisited, J. Austral. Math. Soc. Ser. B, 40 (1999), pp. 353-378. 